\title{
Gonadotrophin-releasing hormone analogue or dienogest plus estradiol valerate to prevent pain recurrence after laparoscopic surgery for endometriosis: a multi-center randomized trial
}

\author{
ROBERTA GRANESE ${ }^{1}$, ANTONINO PERINO², GLORIA CALAGNA ${ }^{2}$, SALVATORE SAITTA ${ }^{3}$, PASQUALE DE \\ FRANCISCIS ${ }^{4}$, NICOLA COLACURCI ${ }^{4}$, ONOFRIO TRIOLO ${ }^{1}$ \& GASPARE CUCINELLA ${ }^{2}$ \\ 'Department of Pediatric, Gynecological, Microbiological and Biomedical Sciences, University Hospital "G. Martino", \\ Messina, ${ }^{2}$ Department of Obstetrics and Gynecology, University Hospital "P. Giaccone", Palermo, ${ }^{3}$ Department of Clinical \\ and Experimental Medicine Human Pathology, University Hospital "G. Martino", Messina, and "Department of Obstetrics \\ and Gynecology, II University, Naples, Italy
}

\section{Key words}

Endometriosis, dienogest, GnRH-analogue, pain recurrence, chronic pelvic pain

\section{Correspondence}

Roberta Granese, Department of Obstetrics and Gynaecology, University Hospital

"Gaetano Martino", v. Consolare Valeria

Gazzi, 98125 Messina, Italy.

E-mail: rgranese@unime.it

\section{Conflict of interest}

The authors have stated explicitly that there are no conflicts of interest in connection with this article.

Please cite this article as: Granese R, Perino A, Calagna G, Saitta S, De Franciscis P, Colacurci $N$ et al. Gonadotrophin-releasing hormone analogue or dienogest plus estradiol valerate to prevent pain recurrence after laparoscopic surgery for endometriosis: a multi-center randomized trial. Acta Obstet Gynecol Scand 2015; DOI: 10.1111/ aogs. 12633

Received: 26 January 2014

Accepted: 4 March 2015

DOI: 10.1111/aogs.12633
Objectives. To evaluate the efficacy of dienogest + estradiol valerate (E2V) and gonadotrophin-releasing hormone analogue (GnRH-a) in reducing recurrence of pain in patients with chronic pelvic pain due to laparoscopically diagnosed and treated endometriosis. Design. Multi-center, prospective, randomized study. Setting. Three university departments of obstetrics and gynecology in Italy. Population. Seventy-eight women who underwent laparoscopic surgery for endometriosis combined with chronic pelvic pain. Methods. Post-operative administration of dienogest $+\mathrm{E} 2 \mathrm{~V}$ for 9 months (group 1) or GnRH-a monthly for 6 months (group 2). Main outcome measures. A visual analogue scale was used to test intensity of pain before laparoscopic surgery at 3,6 and 9 months of follow up. A questionnaire to investigate quality of life was administered before surgery and at 9 months of follow up. Results. The visual analogue scale score did not show any significant differences between the two groups $(p=0.417)$. The questionnaire showed an increase of scores for all women compared with pre-surgery values, demonstrating a marked improvement in quality of life and health-related satisfaction with both treatments. No significant differences were found between the groups. The rate of apparent endometriosis recurrence was $10.8 \%$ in group 1 and $13.7 \%$ in group 2 $(p=0.962)$. Conclusion. Both therapies seemed equally efficacious in preventing endometriosis-related chronic pelvic pain recurrence in the first 9 months of follow-up.

Abbreviations: CA125, cancer antigen 125; CPP, chronic pelvic pain; E2V, estradiol valerate; EHP, Endometriosis Health Profile; GnRH-a, gonadotrophin-releasing hormone analogues; OC, oral contraceptive; QoL, quality of life; VAS, visual analogue scale.

\section{Introduction}

Endometriosis is a chronic disease caused by the growth of functional endometrium-like tissue outside the uterine cavity, which in turn causes infertility and pelvic pain (1). It is a common gynecological disease affecting between 2

\section{Key Message}

Dienogest + estradiol valerate as a back-up therapy for preventing endometriosis-related pain relapse after surgery is as effective as a gonadotrophin-releasing hormone analogue. 
and $10 \%$ of women of reproductive age (2). Painful symptomatology may occur during menstrual bleeding (dysmenorrhea) and sexual intercourse (dyspareunia), or the pain may not follow any cyclical pattern (chronic pelvic pain, CPP) (3). If endometriosis involves the rectum or bladder, dyschezia or dysuria may be present (4). However, the disease is quite complicated and due to this, classification has evolved from a local disorder to a chronic systemic affliction (1). For many patients, it has a severe impact on quality of life (QoL) (5).

Laparoscopic conservative surgery may reduce painful symptoms and improve QoL in $67-80 \%$ of patients (6), but lesions and symptoms frequently recur within 2-5 years $(7,8)$. The likelihood of hospital readmission for endometriosis within 4 years after surgery for additional surgical treatment was found to be $27 \%$ (9), with some women requiring three or more re-operations (10). Repeated surgery is positively associated with increased morbidity and health care costs and, in ovarian endometriosis, with damage to the ovarian reserve (11). Therefore, postoperative medical treatment has been advocated to prolong the symptom-free period and at the same time delay recurrence as long as possible (12,13).

Gonadotrophin-releasing hormone analogues (GnRHa), combined oral contraceptives (OCs) or progestins are the most frequently used hormonal treatment (12). Although effective, most of these options are associated with side-effects, which may affect compliance and preclude long-term use. GnRH-a cause an estrogen-deficient state (14); they also act directly on steroidogenesis and suppress endometrial implant growth (15). After 6 months, additional therapy with OCs is recommended to prevent the loss of bone density becoming a risk factor for fractures (14). When compared with placebo, GnRHa was highly effective in a 6-month trial (16).

Since hormonal therapy is not curative, treatments often need to be administered for years to prolong their effects. For this reason, safe, inexpensive and well-tolerated drugs of known efficacy, such as OCs, were tested (17). In some recent studies, long-term exposure to OCs after conservative surgery for ovarian endometriosis was associated with a major reduction of recurrences $(8,12)$. Moreover, since progesterone is considered the most active drug in the OCs for the prevention of recurrence, new progestins are continually being tested to find one that ensures the desired results with minimal side-effects. Two recent clinical studies in Europe and Japan have investigated dienogest, a 19-nortestosterone derivative, demonstrating that at $2 \mathrm{mg}$ daily, this progestin effectively reduces endometriosis lesions, improves QoL and would appear to be as effective as GnRH-a in terms of endometriosis-related pelvic pain improvement $(18,19)$.
In addition, there are advantages in safety and tolerability, including a reduced incidence of hypoestrogenic effects and minimal change in bone mineral density (5). This drug was marketed initially as an OC, co-administered with estradiol valerate (E2V) (Qlaira ${ }^{\circledR}$, Bayer, Germany). Recently, it has been offered individually for the treatment of endometriosis $(5,20)$.

Based on this, continuous low-dose OCs containing dienogest $+\mathrm{E} 2 \mathrm{~V}$ were tested against GnRH-a in women who had CPP due to pelvic endometriosis after laparoscopic treatment, with the primary outcome to compare efficacy in prevention of pain recurrence and healthrelated QoL for up to 9 months. A secondary outcome was to evaluate the recurrence rate of endometriosis lesions.

\section{Material and methods}

A multi-center prospective randomized study on patients who had undergone conservative surgery for CPP due to endometriosis was conducted in the Departments of Obstetrics and Gynecology of the Universities of Naples, Messina and Palermo, which are all referral centers for the diagnosis and management of endometriotic disease. Permission was obtained from the Local Institutional Review Boards before the study began (approval date 17 July 2009) and each patient gave their signed informed consent for recruitment. Study conduct and analysis was performed following the CONSORT criteria (www.equator-network.org) but the study was not registered in a clinical trials database at the time.

Inclusion criteria were age between 18 and 45 years, no immediate desire for offspring, surgical and histological confirmation of endometriotic disease, a visual analogue scale (VAS, see below) score $>40$ before surgery and no hormone therapy in the 3 months preceding surgery. The exclusion criteria were absence of precise details of the operation or histologic diagnosis of endometriosis, usual contraindications to OCs or GnRH-a, chronic use of alcohol or drugs that affect cognitive function, psychiatric disorders, concomitant neoplastic disease or chronic pelvic inflammatory disease.

Demographic and historical data were collected. A clinical gynecological examination with pelvic and vaginal ultrasound and measurement of serum cancer antigen 125 (CA-125) were performed in all patients before laparoscopic surgery. To assess severity of the symptoms, all patients were invited to score the intensity of pain with a VAS score to evaluate dysmenorrhea, dyspareunia and non-menstrual pelvic pain subjectively on a scale from 0 to $100(0=$ no pain, $100=$ unbearable pain $)$. The VAS score is a validated measure of endometriosis-related pain and is widely used in clinical trials (21). 
In addition, all women completed an Endometriosis Health Profile (EHP) questionnaire, i.e. the short form EHP-5, used to measure effects that endometriosis may have on women's lives, with scores rated on a scale from 0 to 10 (worst health to best health). It is a recommended instrument to assess health status when conducting clinical trials associated with endometriosis (22). Data on the VAS and questionnaire scores are reported as mean \pm standard deviation.

All procedures were performed by expert surgeons (Level II of the Italian Society of Gynecologic Endoscopy). Staging of endometriosis was carried out according to the revised American Fertility Society (rAFS) classification (23). Laparoscopic surgery was performed on all enrolled patients. At histopathological evaluation, samples confirmed the presence of endometriosis in all cases. During laparoscopy, superficial peritoneal endometriosis lesions, ovarian lesions, one bladder nodule, uterosacral ligament nodules, recto-vaginal nodules and all adhesions were identified and removed (Table 1). No bowel resection was performed. No intraoperative complications occurred and no laparotomy conversion was needed.

A random sequence using SPSS version 17.0 (SPSS Inc., Chicago, IL, USA), which returns a random value from a Bernoulli distribution with the specified probability parameter (0.5) was generated. Patients were placed in alphabetical order and then, using the random sequence previously generated, each patient was assigned to one of the two treatment groups, dienogest $+\mathrm{E} 2 \mathrm{~V}$ (group 1, $n=39$ ) or the GnRH-a (group 2, $n=39$ ).

On discharge after laparoscopy, group 1 patients received OCs with dienogest $+\mathrm{E} 2 \mathrm{~V}$ (multiphasic pill with $2 \mathrm{mg}$ of E2V for 22 days $+2 \mathrm{mg}$ of dienogest for 5 days and $3 \mathrm{mg}$ for 17 days; the first two and the last four pills containing only E2V or placebo were removed) (Qlaira ${ }^{\circledR}$, Bayer, Germany). Group 2 patients received $\mathrm{GnRH}-\mathrm{a}$, i.e. leuprorelin acetate $3.75 \mathrm{mg}$, one dose every 30 days
(Enantone $^{\circledR}$, Takeda, Italy). Patients in group 1 repeated this administration of OCs without interruption for 9 months. In group 2, the GnRH-a dose was repeated every 30 days for 6 months.

Follow-up visits were at 3, 6 and 9 months after treatment. At each follow-up, clinical assessment, vaginal and rectal examination and transvaginal ultrasonography were performed to evaluate potential endometriosis recurrence and VAS score assessed to evaluate the presence or severity of pelvic pain. Recurrence was defined, for convenience, as the presence of an endometriosis lesion, visible with transvaginal ultrasound scanning or felt at vaginal examination. Highly qualified sonographers with extensive experience in the detection of endometriotic lesions performed all scans. We defined ovarian endometriotic cysts according to the diagnostic ultrasound criteria established by Mais et al. (24).

At the 9-month follow up, the serum level of CA-125 was measured and patients' QoL tested using the same questionnaire as in the pretreatment phase.

\section{Statistical analysis}

Statistical analysis was performed with SPSS for Windows. Differences between two data series were analyzed by the Mann-Whitney test, and differences between categorical groups by Fisher's exact test. A log-rank test was performed to assess differences in rate of recurrent endometriosis between different treatments. Statistical significance was set at $p<0.05$. At alpha $=0.05$, the study power was $80 \%$ with a difference of $7 \pm 11$ (OC therapy) and $16 \pm 20$ (GnRH-a therapy) in the mean VAS score.

\section{Results}

An overview of the study is shown in Figure 1 in a flow diagram. Characteristics of the patients at baseline are

Table 1. Laparoscopic surgical procedures among the randomized women.

\begin{tabular}{|c|c|c|c|c|c|}
\hline \multirow[b]{2}{*}{ Laparoscopic surgery } & \multicolumn{2}{|c|}{ Group $1(n=39)$} & \multicolumn{2}{|c|}{ Group $2(n=39)$} & \multirow[b]{2}{*}{$p$-value } \\
\hline & $n$ & $\%$ & $n$ & $\%$ & \\
\hline Unilateral cystectomy & 19 & 48.7 & 22 & 56.4 & 0.65 \\
\hline Bilateral cystectomy & 10 & 25.6 & 7 & 17.9 & 0.584 \\
\hline Rectovaginal nodule removal & 2 & 5.1 & 1 & 2.6 & 1 \\
\hline Uterosacral ligament nodule removal & 2 & 5.1 & 4 & 10.3 & 0.675 \\
\hline Bladder nodule removal & 0 & 0 & 1 & 2.6 & 1 \\
\hline Ablation of peritoneal endometriosis & 33 & 84.6 & 35 & 89.7 & 0.737 \\
\hline Thin and tough adhesion removal & 36 & 92.3 & 38 & 97.4 & 0.615 \\
\hline Unilateral salpingoophorectomy & 4 & 10.3 & 3 & 7.7 & 1 \\
\hline Bilateral salpingoophorectomy & 0 & & 1 & 2.6 & 1 \\
\hline
\end{tabular}

Group 1: dienogest + estradiol valerate (E2V); Group 2: leuprorelin acetate (GnRH-a). Statistical analysis was performed using Fisher's exact test. 


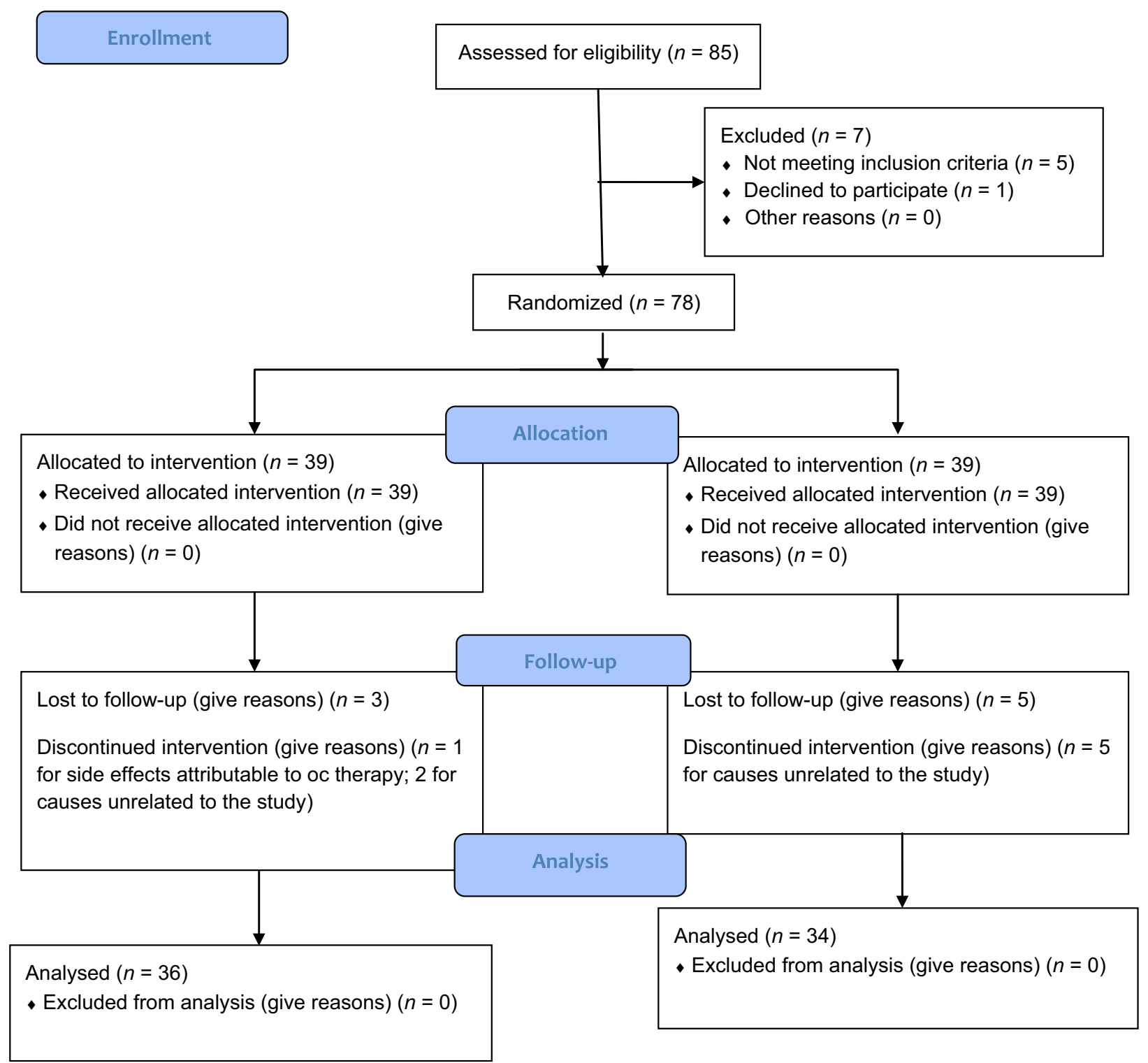

Figure 1. CONSORT flow diagram showing a study overview.

reported in Table 2. There were no significant differences between the groups in terms of age $(p=0.532)$, body mass index $(p=0.398)$, AFS stages $(p=0.847)$, VAS scores $(p=0.269)$ or CA-125 $(p=0.32)$ before treatment. Three patients $(7.7 \%)$ in group 1 and five $(12.8 \%)$ in group 2 did not complete the study: seven for causes unrelated to the study and one for side-effects attributable to OC therapy.

Visual analogue scale data did not show significant differences in any of the follow-up visits between the two groups $(p=0.417)$ (Figure 2) and were lower in the first follow up than before surgery in both group 1 $(p<0.0001)$ and group $2(p<0.001)$.
Recurrent disease was represented in all cases by an ovarian cyst, unilateral in five cases and bilateral in only one case (Table 3). The ultrasound characteristics of these cysts were typical of endometrioma (unilocular, with a regular profile, "ground glass" appearance, with a thick wall and absence of internal papillary projections) $(24,25)$. The mean size of the cysts was $2.6( \pm 0.6) \mathrm{cm}$. In only two cases were the cysts associated with pelvic pain. We could not exclude misdiagnosis of minimal endometriotic foci as these are not detectable by ultrasound or by vaginal gynecologic exploration. No sign of endometriosis was present at the first follow up in any of the women who subsequently reported a recurrence of the disease. 
Table 2. Demographic characteristics of the patients at baseline.

\begin{tabular}{lccc}
\hline & $\begin{array}{c}\text { Group 1 }(n=39) \\
\text { Mean } \pm \text { SD }\end{array}$ & $\begin{array}{l}\text { Group 2 }(n=39) \\
\text { Mean } \pm \text { SD }\end{array}$ & $p$-value \\
\hline Age (years) & $31.2 \pm 2.6$ & $30.5 \pm 2.5$ & 0.532 \\
BMI $\left(\mathrm{kg} / \mathrm{m}^{2}\right)$ & $21.7 \pm 2.3$ & $22.4 \pm 2.1$ & 0.398 \\
rAFS stage $(n)$ & & & \\
I & 2 & 3 & 0.847 \\
II & 6 & 20 & \\
III & 23 & 9 & \\
IV & 8 & $53.6 \pm 20.2$ & 0.269 \\
VAS score & $48.4 \pm 18.7$ & $89 \pm 28.6$ & 0.32 \\
CA-125 $(\mathrm{U} / \mathrm{mL})$ & $97.8 \pm 31.4$ & & \\
\hline
\end{tabular}

All data are expressed as mean \pm SD except AFS stage, which is expressed in number of patients (n). Statistical analysis was performed using the Mann-Whitney test. Group 1: dienogest + estradiol valerate (E2V); Group 2: leuprorelin acetate ( $\mathrm{GnRH}-\mathrm{a})$.

BMI, body mass index; rAFS, Revised American Fertility Society Score.

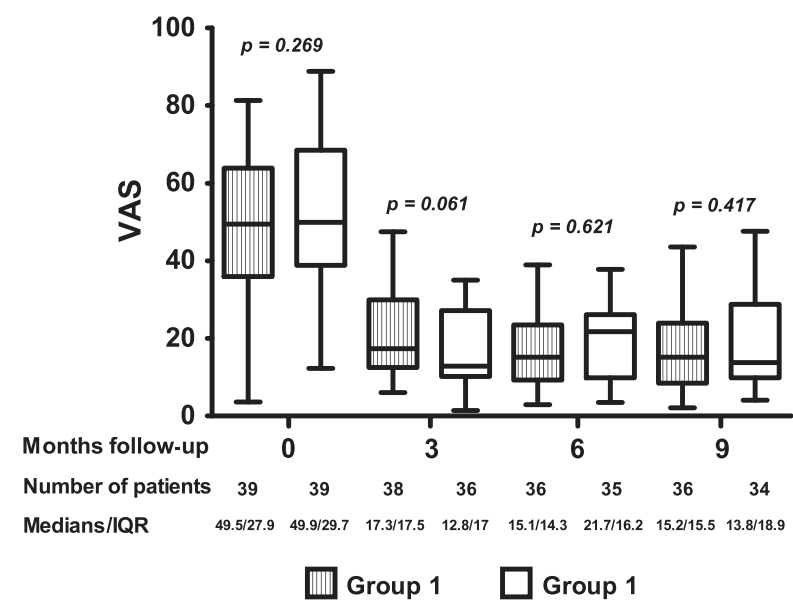

Figure 2. Comparison of visual analogue scale (VAS) scores in group 1 (dienogest + estradiol valerate) and group 2 (leuprorelin acetate $3.75 \mathrm{mg}$ ). IQR, interquartile range.

For this reason, we considered the cyst a "recurrence" and not "a persistence" of the disease.

Rates of cumulative recurrence were $10.8 \%$ in group 1 and $13.7 \%$ in group 2, but this difference was not statistically significant between the groups $(p=0.962)$. Endometriosis recurrence and side-effects at each follow-up are reported in Table 3.

At the 9-month follow up, the questionnaire results showed a considerable increase of scores for all women compared with before surgery, demonstrating an improvement in the QoL and an equal health-related satisfaction with both treatments. In group 1 , the mean score before treatment was $4.2 \pm 1.9$ and after treatment $8.6 \pm 2$; in group 2 the mean score before treatment was $4.9 \pm 2$ and after treatment $9.1 \pm 1.8$. No differences were found between the two groups at baseline and at 9-month follow up, respectively ( $p=0.123$ and $p=0.391$ ). CA-125 mean value at 9 months was $22.3 \pm 16.9$ in group 1 and $25.1 \pm 19.7$ in group $2(p=0.541)$.

\section{Discussion}

The current study was an attempt to find the best solution, in terms of efficacy, to prevent and/or decrease the risk of CPP due to pelvic endometriosis after laparoscopic treatment, comparing the effect of OCs with dienogest and a GnRH-a. The study also evaluated the recurrence rate of endometriosis lesions.

Approximately $40-45 \%$ of patients have a relapse of the disease that engenders further surgery (26). There is worldwide concern regarding the risk of repeated operations for an endometrioma as a major cause of ovarian insufficiency (27). For this reason, it is important to base post-surgery therapeutic strategies on factors which affect the natural course of the disease and its tendency to reappear (7).

The American Congress of Obstetricians and Gynecologists Practice Bulletin suggests that the true recurrence rate must be even higher than the rate generally stated, as most publications report symptomatic disease only (28). In addition, there is much variance in the reported cumulative recurrence rate among published studies. The definition of recurrence also varies (subjective feeling of pain or more objective clinical/instrumental measures) with type of endometriosis, methods of surgery, disease severity, and surgeon's skills being among the known or potential factors that may influence recurrence risk.

The rationale for postoperative treatment is to perform complete eradication of the lesions that could not be surgically removed, or to treat microscopic foci that may not have been evident to the surgeon, as well as to prevent iatrogenic dissemination of endometriotic cells (29). But as medical treatment is suppressive and not extirpative therapy, pain may recur after discontinuation. Therefore, long-term treatment is needed and consideration has been given not only to efficacy, but also to the safety and tolerability of the treatment options available.

The European Society of Human Reproduction and Embryology guidelines recommend suppression of ovarian function with hormonal therapy with level A-B evidence (30). Gonadotrophin-releasing hormone analogues combined OCs or progestins are the options most usually adopted. Vast differences in treatment modality, types of endometriosis, length of follow up and outcome measurements preclude a meaningful systematic review of their use.

GnRH-a have long been used in the treatment of CPP related to endometriosis, and administration for 6 months 
Table 3. Endometriosis recurrence and side-effects at 3, 6 and 9 months of follow up.

\begin{tabular}{|c|c|c|c|c|c|c|c|c|c|c|c|c|c|c|c|}
\hline \multirow[b]{3}{*}{ Endometriosis recurrence } & \multicolumn{5}{|c|}{ 3-month follow up } & \multicolumn{5}{|c|}{ 6-month follow up } & \multicolumn{5}{|c|}{ 9-month follow up } \\
\hline & \multicolumn{2}{|c|}{$\begin{array}{l}\text { Group } 1 \\
(n=39)\end{array}$} & \multicolumn{2}{|c|}{$\begin{array}{l}\text { Group } 2 \\
(n=39)\end{array}$} & \multirow[b]{2}{*}{$p$} & \multicolumn{2}{|c|}{$\begin{array}{l}\text { Group } 1 \\
(n=36)\end{array}$} & \multicolumn{2}{|c|}{$\begin{array}{l}\text { Group } 2 \\
(n=35)\end{array}$} & \multirow[b]{2}{*}{$p$} & \multicolumn{2}{|c|}{$\begin{array}{l}\text { Group } 1 \\
(n=36)\end{array}$} & \multicolumn{2}{|c|}{$\begin{array}{l}\text { Group } \\
2 \\
(n=34)\end{array}$} & \multirow[b]{2}{*}{$p$} \\
\hline & $n$ & $\%$ & $n$ & $\%$ & & $n$ & $\%$ & $n$ & $\%$ & & $n$ & $\%$ & $n$ & $\%$ & \\
\hline Unilateral cyst & \multicolumn{2}{|c|}{-} & \multicolumn{2}{|c|}{-} & - & \multicolumn{2}{|l|}{1} & \multicolumn{2}{|l|}{1} & 1 & \multicolumn{2}{|l|}{2} & \multicolumn{2}{|l|}{1} & 1 \\
\hline Bilateral cyst & \multicolumn{2}{|c|}{ - } & \multicolumn{2}{|c|}{-} & - & \multicolumn{2}{|c|}{-} & \multicolumn{2}{|c|}{-} & - & \multicolumn{2}{|c|}{ - } & \multicolumn{2}{|l|}{1} & 0.486 \\
\hline Other recurrence & \multicolumn{2}{|c|}{ - } & \multicolumn{2}{|c|}{-} & - & \multicolumn{2}{|c|}{-} & \multicolumn{2}{|c|}{-} & - & \multirow{2}{*}{\multicolumn{2}{|c|}{-}} & \multicolumn{2}{|c|}{-} & - \\
\hline \multicolumn{14}{|l|}{ Side-effects } & & \\
\hline Headache & 6 & 15 & 4 & 10 & 0.737 & 7 & 19 & 6 & 17 & 1 & 7 & 19 & 1 & 3 & 0.056 \\
\hline Decreased libido & 10 & 26 & 7 & 18 & 0.584 & 11 & 31 & 9 & 26 & 0.793 & 12 & 33 & 4 & 12 & 0.046 \\
\hline Spotting & 6 & 15 & 2 & 5 & 0.263 & 5 & 14 & 1 & 3 & 0.199 & 2 & 6 & 0 & 0 & 0.493 \\
\hline Vaginal dryness & 5 & 13 & 8 & 21 & 0.545 & 7 & 19 & 10 & 29 & 0.415 & 8 & 22 & 1 & 3 & 0.028 \\
\hline Vasomotor symptoms & 0 & 0 & 7 & 18 & 0.012 & 0 & 0 & 10 & 29 & $<0.0001$ & 0 & 0 & 1 & 3 & 0.486 \\
\hline Discomfort from amenorrhea & 10 & 26 & 15 & 38 & 0.332 & 10 & 28 & 17 & 49 & 0.09 & 10 & 28 & 0 & 0 & 0.001 \\
\hline Weight gain & 1 & 3 & 2 & 5 & 1 & 2 & 6 & 4 & 11 & 0.429 & 2 & 6 & 1 & 3 & 1 \\
\hline
\end{tabular}

Group 1: dienogest + estradiol valerate (E2V); Group 2: leuprorelin acetate (GnRH-a). Statistical analysis was performed using Fisher's exact test.

(which is the maximum recommended treatment duration when no estrogen add-back therapy is used) appears to improve the duration of pain relief (14). In our study we compared GnRH-a to OCs containing dienogest and we found a similar effectiveness in reducing pelvic pain associated with endometriosis up to 9 months of follow up, although long-term GnRH-a tolerability would be unlikely if the treatment were to be continued for longer (14). Preoperative characteristics of the patients had no significant influence on subsequent pain improvement. Pain was evaluated with a VAS score, one of the most utilized tests in clinical research even if it is not fully objective (31); it has, however, the advantage of simplicity, is languageindependent and is easily understood by most patients. The VAS score was assessed at the beginning of the study and again at all follow-up visits; there was a considerable increase of scores and thus a marked improvement in QoL and health-related satisfaction with both treatments. We used a short form of the EHP questionnaire which has proved to be useful in a previous Italian study. However, it must be kept in mind that the Italian version of EHP-5 has not been validated and therefore data obtained with it cannot be compared with data obtained with validated EHP-5 results in other languages, such as the original British version (32). During administration of the two hormonal therapies, at the sixth month of follow up we found GnRH-a and the dienogest OCs to be similar with regard to the side-effects of headache, vaginal dryness and weight gain. The only significant differences were in vasomotor symptoms, decreased libido and discomfort due to amenorrhea. Prolonged administration of GnRH-a is limited by undesired effects on bone mineral density and climacteric symptoms. In this perspective, continuous OCs are probably the better choice to prevent effects of estrogen deprivation. The effects of surgery and post-surgical medical treatment could not be dissociated from the study design, but increased patient satisfaction can be regarded as important and may lead to better treatment compliance in the long run.

A randomized comparative trial showed that postoperative treatment after surgery for pelvic endometriosis, either with GnRH-a or with continuous monophasic OCs, at an early follow up of 12 months was more effective than placebo in terms of relief of endometriosis-associated pain. No data were available on recurrence of asymptomatic disease (33). In addition, in another randomized multi-center trial, GnRH-a and dienogest demonstrated the same significant improvements of pelvic pain due to endometriosis at 6 months of follow up (5). Unfortunately, although effective, GnRH-a therapy is expensive and associated with side-effects (16) due to the prolonged hypoestrogenic state it causes. Therefore, longterm use, in the absence of add-back therapy, is precluded.

Oral contraceptives can provide a better option in terms of safety, tolerability and cost, and can be administered for long periods $(8,12)$. They may induce atrophy of the peritoneal endometriotic implants (34) and reduce diseaserelated pain (35). Moreover, OCs reduce retrograde menstrual bleeding and inhibit ovulation, both of which are considered etiopathogenetic factors for endometriosis $(7,8)$. Progestogens contained in OCs also prevent implantation and growth of regurgitated endometrium by inhibiting matrix metalloproteinase expression and angiogenesis, which reduce the inflammatory state and immune response (36). All these reported mechanisms have been analyzed in 
recent studies that appraised the validity of long-term administration of OCs in preventing ovarian endometrioma (12,37) and the recurrence of painful symptoms (38). Vercellini et al. reported that patients who always used OCs had an absolute risk reduction of recurrence of $47 \%$ when compared with never users (8). No differences between available formulations were found among the various kinds of OCs $(12,39)$. A comparative study between postoperative administration of OCs containing dienogest or a levonorgestrel intrauterine system (LNG-IUS) for prevention of pain relapse and disease recurrence in endometriosis patients, demonstrated better efficacy of OCs in reducing pelvic pain at 12 months of follow up (2).

The progestogen component of the "combined pill" is widely considered the active therapeutic drug in treating or preventing endometriosis (12). In recent years, the attention of clinicians has been focused on dienogest, which is currently the only steroidal treatment marketed in most of Europe. Dienogest reduces endometriotic lesions by creating a local progestogenic environment, suppressing the systemic estrogen level moderately (32). Some studies have demonstrated that it is affective as GnRH-a therapy in terms of endometriosis-related pelvic pain improvement (19) and also offers advantages in terms of safety and tolerability (5). The reasoning behind using an estradiol-based combined pill in our study, when endometriosis is clearly an estrogen-sensitive disease, is that it also provides contraception, which, officially, dienogest does not. Furthermore, the control cycle is sometimes better, with less spotting. It is fair to point out that endometriosis benefits from non-fluctuating hormonal situations, such as pregnancy, so we assumed that the presence of a small amount of estrogen was not harmful.

Recurrence has been defined in varying ways in many published studies, making it difficult to estimate recurrence rates. Vignali et al. (40) reported that 3- and 5-year recurrence rates were 20.5 and $43.5 \%$, respectively, for pain, but the corresponding rates for clinical recurrence were 9 and 28\% (40). Overall, it appears that recurrence rates for pain or dysmenorrhea are higher than for clinical recurrences as defined by more objective and verifiable criteria such as sonography. We found a $10-15 \%$ rate of recurrence of endometriosis lesions at 9 months of follow up but with no significant differences between the two treatment modules used, in spite of GnRH-a administration for only a 6-month period and OCs for 9 months. This circumstance might be explained by the effect of GnRH-a on endometriosis, which may last up to several months after suspension (38). In only two cases was the recurrence of an endometrioma associated with pelvic pain, thus confirming that having a detectable clinical recurrence is not a prerequisite for the presence of symptoms, and that symptoms may precede or succeed clinical recurrence.

A potential bias of this study was that women were only followed to 9 months, since long-term recurrence of endometriosis is possible, but data are lacking on longterm symptom profiles. Based on our study results we consider dienogest $+\mathrm{E} 2 \mathrm{~V}$ a valid therapy for preventing endometriosis-related pain relapse after surgery, with an efficacy similar to that of GnRH-a.

\section{Funding}

No special funding.

\section{References}

1. Gentilini D, Perino A, Viganò P, Chiodo I, Cucinella G, Vignali M, et al. Gene expression profiling of peripheral blood mononuclear cells in endometriosis identifies genes altered in non-gynaecologic chronic inflammatory diseases. Hum Reprod. 2011;26:3109-17.

2. Morelli M, Sacchinelli A, Venturella R, Mocciaro R, Zullo F. Postoperative administration of dienogest plus estradiol valerate versus levonorgestrel-releasing intrauterine device for prevention of pain relapse and disease recurrence in endometriosis patients. J Obstet Gynaecol Res. 2013;39:985-90.

3. Fauconnier A, Chapron C. Endometriosis and pelvic pain: epidemiological evidence of the relationship and implications. Hum Reprod Update. 2005;11:595-606.

4. Granese R, Candiani M, Perino A, Venezia R, Cucinella G. Bladder endometriosis: laparoscopic treatment and followup. Eur J Obstet Gynecol Reprod Biol. 2008;140:114-7.

5. Strowitzki T, Marr J, Gerlinger C, Faustmann T, Seitz C. Dienogest is as effective as leuprolide acetate in treating the painful symptoms of endometriosis: a 24-week, randomized, multicentre, open-label trial. Hum Reprod. 2010;25:633-41.

6. Muzii L, Marana R, Angioli R, Bianchi A, Cucinella G, Vignali $\mathrm{M}$, et al. Histologic analysis of specimens from laparoscopic endometrioma excision performed by different surgeons: does the surgeon matter? Fertil Steril. 2011;95:2116-9.

7. Vercellini $\mathrm{P}$, Somigliana E, Viganò $\mathrm{P}$, De Matteis $\mathrm{S}$, Barbara G, Fedele L. Post-operative endometriosis recurrence: a plea for prevention based on pathogenetic, epidemiological and clinical evidence. Reprod Biomed Online. 2010;21:259-65.

8. Vercellini P, Somigliana E, Daguati R, Vigano P, Meroni F, Crosignani PG. Post-operative oral contraceptive exposure and risk of endometrioma recurrence. Am J Obstet Gynecol. 2008;198:504.e1-5.

9. Weir E, Mustard C, Cohen M, Kung R. Endometriosis: what is the risk of hospital admission, readmission, and 
major surgical intervention? J Minim Invasive Gynecol. 2005;12:486-93.

10. Cheong Y, Tay P, Luk F, Gan HC, Li TC, Cooke I. Laparoscopic surgery for endometriosis: how often do we need to re-operate? J Obstet Gynaecol. 2008;28:82-5.

11. Hachisuga T, Kawarabayashi T. Histopathological analysis of laparoscopically treated ovarian endometriotic cysts with special reference to loss of follicles. Hum Reprod. 2002;17:432-5.

12. Cucinella G, Granese R, Calagna G, Svelato A, Saitta S, Tonni G, et al. Oral contraceptives in the prevention of endometrioma recurrence: does the different progestins used make a difference? Arch Gynecol Obstet. 2013;288:821-7.

13. Donnez J, Pirard C, Smets M, Jadoul P, Squifflet J. Surgical management of endometriosis. Best Pract Res Clin Obstet Gynaecol. 2004;18:329-48.

14. Batzer FR. GnRH analogs: options for endometriosisassociated pain treatment. J Minim Invasive Gynecol. 2006;13:539-45.

15. Meresman GF, Bilotas MA, Lombardi E, Tesone M, Sueldo C, Baranao RI. Effect of GnRH analogues on apoptosis and release of interleukin-1b and vascular endothelial growth factor in endometrial cell cultures from patients with endometriosis. Hum Reprod. 2005;18:1767-71.

16. Dlugi AM, Miller JD, Knittle J. Lupron depot (leuprolide acetate for depot suspension) in the treatment of endometriosis: a randomized, placebo-controlled, doubleblind study. Lupron Study Group. Fertil Steril. 1990;54:419-27.

17. Parazzini F, Di Cintio E, Chatenoud L, Moroni S, Ardovino I, Struzziero E, et al. Estroprogestin vs. gonadotrophin agonists plus estroprogestin in the treatment of endometriosis-related pelvic pain: a randomized trial. Eur J Obstet Gynecol Reprod Biol. 2000;88:11-4.

18. Schindler AE. Dienogest in long-term treatment of endometriosis. Int J Womens Health. 2011;3:175-84.

19. McCormack PL. Dienogest: a review of its use in the treatment of endometriosis. Drugs. 2010;70:2073-88.

20. Petraglia F, Hornung D, Seitz C, Faustmann T, Gerlinger C, Luisi S, et al. Reduced pelvic pain in women with endometriosis: efficacy of long-term dienogest treatment. Arch Gynecol Obstet. 2012;285:167-73.

21. Gerlinger C, Schumacher U, Faustmann T, Colligs A, Schmitz H, Seitz C. Defining a minimal clinically important difference for endometriosis-associated pelvic pain measured on a visual analog scale: analyses of two placebo-controlled, randomized trials. Health Qual Life Outcomes. 2010;8:13.

22. Practice Committee of American Society for Reproductive Medicine. Treatment of pelvic pain associated with endometriosis. Fertil Steril. 2008;90(5 Suppl.):S260-9.

23. Revised American Society for Reproductive Medicine classification of endometriosis: 1996. Fertil Steril. 1997;67:817-21.
24. Mais V, Guerriero S, Ajossa S, Angiolucci M, Paoletti AM, Melis GB. The efficiency of transvaginal ultrasonography in the diagnosis of endometrioma. Fertil Steril. 1993;60:776-80.

25. Savelli L. Transvaginal sonography for the assessment of ovarian and pelvic endometriosis: how deep is our understanding? Ultrasound Obstet Gynecol. 2009;33:497501.

26. Garry R. The effectiveness of laparoscopic excision of endometriosis. Curr Opin Obstet Gynecol. 2004;16:299-303.

27. Takae S, Kawamura K, Sato Y, Nishijima C, Yoshioka N, Sugishita Y, et al. Analysis of late-onset ovarian insufficiency after ovarian surgery: retrospective study with 75 patients of post-surgical ovarian insufficiency. PLoS ONE. 2014;23:e98174.

28. ACOG Committee on Practice Bulletins-Gynaecology. ACOG practice bulletin. Medical management of endometriosis. Number 11, December 1999 (replaces technical bulletin number 184, September 1993). Clinical management guidelines for obstetrician-gynecologists. Int J Gynaecol Obstet. 2000;71:183-96.

29. Thomas EJ. Combining medical and surgical treatment for endometriosis: the best of both worlds? BJOG. 1992;99 (Suppl. 7):5-8.

30. Dunselman GA, Vermeulen N, Becker C, Calhaz-Jorge C, D'Hooghe T, De Bie B, et al. ESHRE guideline: management of women with endometriosis. Hum Reprod. 2014;29:400-12.

31. Oettel M, Breitbarth H, Elger W, Gräser T, Hübler D, Kaufmann G, et al. The pharmacological profile of dienogest. Eur J Contracept Reprod Health Care. 1999;4(1 Suppl.):2-13.

32. Vercellini P, Frattaruolo MP, Somigliana E, Jones GL, Consonni D, Alberico D, et al. Surgical versus low-dose progestin treatment for endometriosis-associated severe deep dyspareunia II: effect on sexual functioning, psychological status and health-related quality of life. Hum Reprod. 2013;28:1221-30.

33. Sesti F, Pietropolli A, Capozzolo T, Broccoli P, Pierangeli S, Bollea MR, et al. Hormonal suppression treatment or dietary therapy versus placebo in the control of painful symptoms after conservative surgery for endometriosis stage III-IV. A randomized comparative trial. Fertil Steril. 2007;88:1541-7.

34. Rodgers AK, Falcone T. Treatment strategies for endometriosis. Expert Opin Pharmacother. 2008;9:243-55.

35. Vercellini P, Fedele L, Pietropaolo G, Frontino G, Somigliana E, Crosignani PG. Progestogens for endometriosis: forward to the past. Hum Reprod Update. 2003;9:387-96.

36. Seracchioli R, Mabrouk M, Frascà C, Manuzzi L, Savelli L, Venturoli S. Long-term oralcontraceptive pills and postoperativepain management after laparoscopic excision of ovarian endometrioma: a randomized controlled trial. Fertil Steril. 2010;94:464-71. 
37. Vercellini P, Crosignani PG, Fadini R, Radici E, Belloni C, Sismondi P. A gonadotrophin-releasing hormone agonist compared with expectant management after conservative surgery for symptomatic endometriosis. BJOG.

1999;106:672-7.

38. Bayoglu Tekin Y, Dilbaz B, Altinbas SK, Dilbaz S. Postoperative medical treatment of chronic pelvic pain related to severe endometriosis: levonorgestrel-releasing intrauterine system versus gonadotropin-releasing hormone analogue. Fertil Steril. 2011;95:492-6.

39. Davis L, Kennedy SS, Moore J, Prentice A. Modern combined oral contraceptives for pain associated with endometriosis. Cochrane Database Syst Rev. 2007;3:CD001019.

40. Vignali M, Bianchi S, Candiani M, Spadaccini G, Oggioni G, Busacca M. Surgical treatment of deep endometriosis and risk of recurrence. J Minim Invasive Gynecol. 2005;12:508-13. 